\title{
The IFMIF-EVEDA accelerator beam dump design
}

\author{
D. Iglesias ${ }^{a}$, F. Arranz ${ }^{a}$, J.M. Arroyo ${ }^{a}$, G. Barrera ${ }^{a}$, B. Brañas ${ }^{a, *}$, N. Casal ${ }^{a}$, M. García ${ }^{b . c}$, D. López ${ }^{\text {b.c. }}$, \\ J.I. Martínez ${ }^{a}$, A. Mayoral ${ }^{b, c}$, F. Ogando ${ }^{b, c}$, M. Parro ${ }^{a}$, C. Oliver ${ }^{a}$, D. Rapisarda ${ }^{a}$, J. Sanz ${ }^{b . c}$, P. Sauvan ${ }^{b . c}$, \\ A. Ibarra ${ }^{a}$ \\ a CIEMAT, Avda. Complutense 22,28040 Madid, Spain \\ bDpto. Ingenieria Energética, UNED, 28040 Madrid, Spain \\ "Instituto de Fusión Nuclear, UPM, 28040 Madrid, Spain
}

\section{A R T I C L E I N F O}

Article history:

Available online $x x x x$

\begin{abstract}
A B S T R A C T
The IFMIF-EVEDA accelerator will be a $9 \mathrm{MeV}, 125 \mathrm{~mA} \mathrm{cw}$ deuteron accelerator prototype for verifying the validity of the $40 \mathrm{MeV}$ accelerator design for IFMIF. A beam dump designed for maximum power of $1.12 \mathrm{MW}$ will be used to stop the beam at the accelerator exit. The conceptual design for the IFMIFEVEDA accelerator beam dump is based on a conical beam stop made of OFE copper. The cooling system uses an axial high velocity flow of water pressurized up to $3.4 \times 10^{5} \mathrm{~Pa}$ to avoid boiling. The design has been shown to be compliant with ASME mechanical design rules under nominal full power conditions. A sensitivity analysis has been performed to take into account the possible margin on the beam properties at the beam dump entrance. This analysis together with the study of the maintenance issues and the mounting and dismounting operations has led to the complete design definition.
\end{abstract}

\section{Introduction}

The IFMIF-EVEDA accelerator [1] will be a $9 \mathrm{MeV}, 125 \mathrm{~mA} \mathrm{cw}$ deuteron accelerator, identical to the low energy section of one of the $40 \mathrm{MeV}$ IFMIF accelerators, which will be tested to verify the validity of the design before launching the IFMIF construction. It includes an ion source, a Radiofrequency Quadrupole cavity and the first module of a superconducting linac based on half wave resonator cavities. As no target is planned for the accelerated beam, a beam dump is required for the commissioning and accelerator tests.

To minimize the accelerator activation, the commissioning will be performed mainly with $\mathrm{H}_{2}^{+}$pulsed beams with progressively increasing duty factor and current up to the nominal $125 \mathrm{~mA}$ current. Therefore the beam dump must be able to stop $\mathrm{D}^{+}$and $\mathrm{H}_{2}^{+}$ continuous and pulsed beams with energies $5 \mathrm{MeV}$ (RFQ commissioning) and $9 \mathrm{MeV}$. The maximum beam power is $1.12 \mathrm{MW}$, corresponding to a beam current of $125 \mathrm{~mA}$. A maximum operation time at full current of $\mathrm{D}^{+}$of around 1 year and a 2 months operation time with $5 \mathrm{~Hz}$ pulsed beam with $0.1 \%$ duty cycle have been assumed. The design must be such that the following requirements are fulfilled:
- Dose values outside the vault during accelerator operation must be below the acceptable levels for workers. Doses of $12.5 \mu \mathrm{Sv} / \mathrm{h}$ are acceptable inside the vault.

- Personnel access to the accelerator vault must be allowed for maintenance during beam-off phases.

At the end of the EVEDA tests, the beam dump must qualify for a suitable waste management option such as shallow burial or near surface disposal. The conceptual design is based on the LEDA beam dump concept, which proved to be very robust during operation. The main difference is the material chosen, Copper in this case because of its thermal properties and activation criteria. Also, the properties of the IFMIF-EVEDA beam make a pure conical shape better suited than the ogive shape used in LEDA [9].

\section{Mechanical design}

\subsection{Beam shape and geometry of beam-facing wall}

The temperature, thermal gradients and stress fields in the beam dump material are directly dependant on the power density profile [2]. As the power density increases, the design of the beam dump becomes more complex. Therefore it is important to reduce the maximum deposited power density as much as possible. Power densities between 2 and $3 \mathrm{MW} / \mathrm{m}^{2}$ have been found acceptable for actual design and materials, depending on the place where they are located. The reduction of this power density is achieved by 

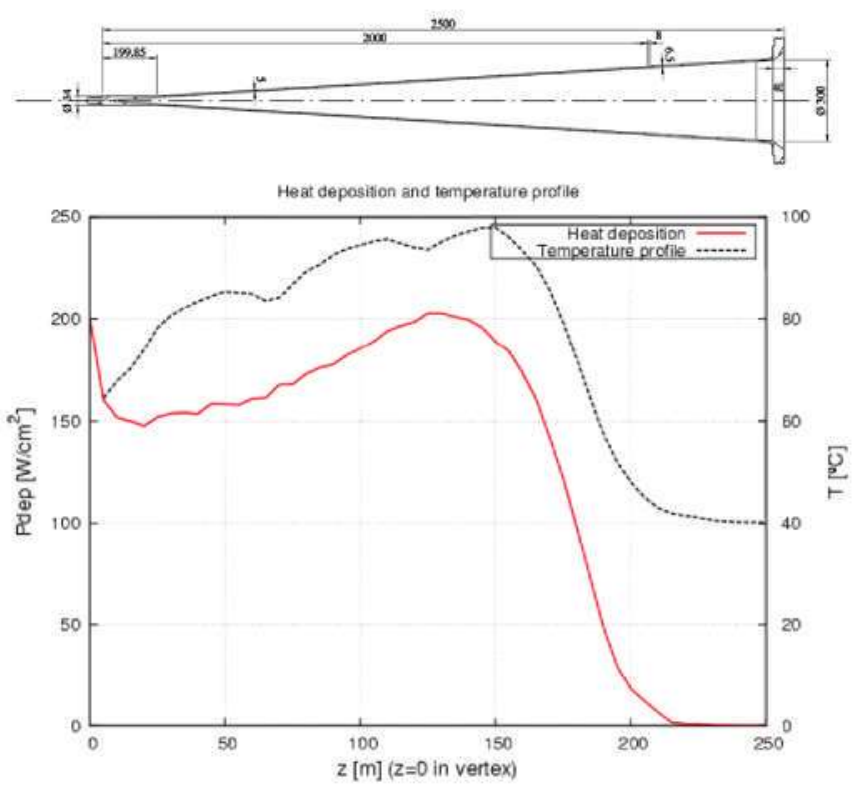

Fig. 1. Internal cone geometry, power density and temperature profiles.

defocusing the beam to increase its size and by using a very low incidence angle in the beam stop thus maximizing the material surface area hit by the beam.

The beam, which has an rms size of $3 \mathrm{~mm}$ at the accelerator exit, is expanded by the action of three quadrupoles whose currents and positions have been chosen to obtain the highest possible beam size $(40 \mathrm{~mm})$ and divergence $(15.8 \& 17 \mathrm{mrad})$ at the beam dump entrance. The resultant beam shape at this location departs from a Gaussian, being less peaked and with less power at the edges. It is almost circular at beam dump entrance but, as divergence values are different, its shape is progressively transformed to an ellipse.

A conical geometry was selected for the beam facing surface (Fig. 1). This shape is adequate for the almost axis-symmetric IFMIF-EVEDA beam [3]. The resulting beam power deposition profile (Fig. 1, lower part) is calculated from particle simulations [4], taking into account the effect of backscattered ions, computed by the SRIM code [5].

Previous experiences and the space availability led to a length choice of $2.5 \mathrm{~m}$. The cone diameter must be large enough to contain the whole beam including the halo (for Gaussian beams, an aperture of five times the rms size is normally used). As the initial cone diameter is fixed at $300 \mathrm{~mm}$, a chamfer located at the entrance is included to absorb the halo power deposition.

\subsection{Cooling system}

The coolant channel geometry is chosen to provide adequate velocity in the high power density zone (from the cone tip to the middle of the beam dump), avoiding high values, which would produce vibrations and material erosion, and too low values, which would not provide enough heat transfer. The cooling water enters

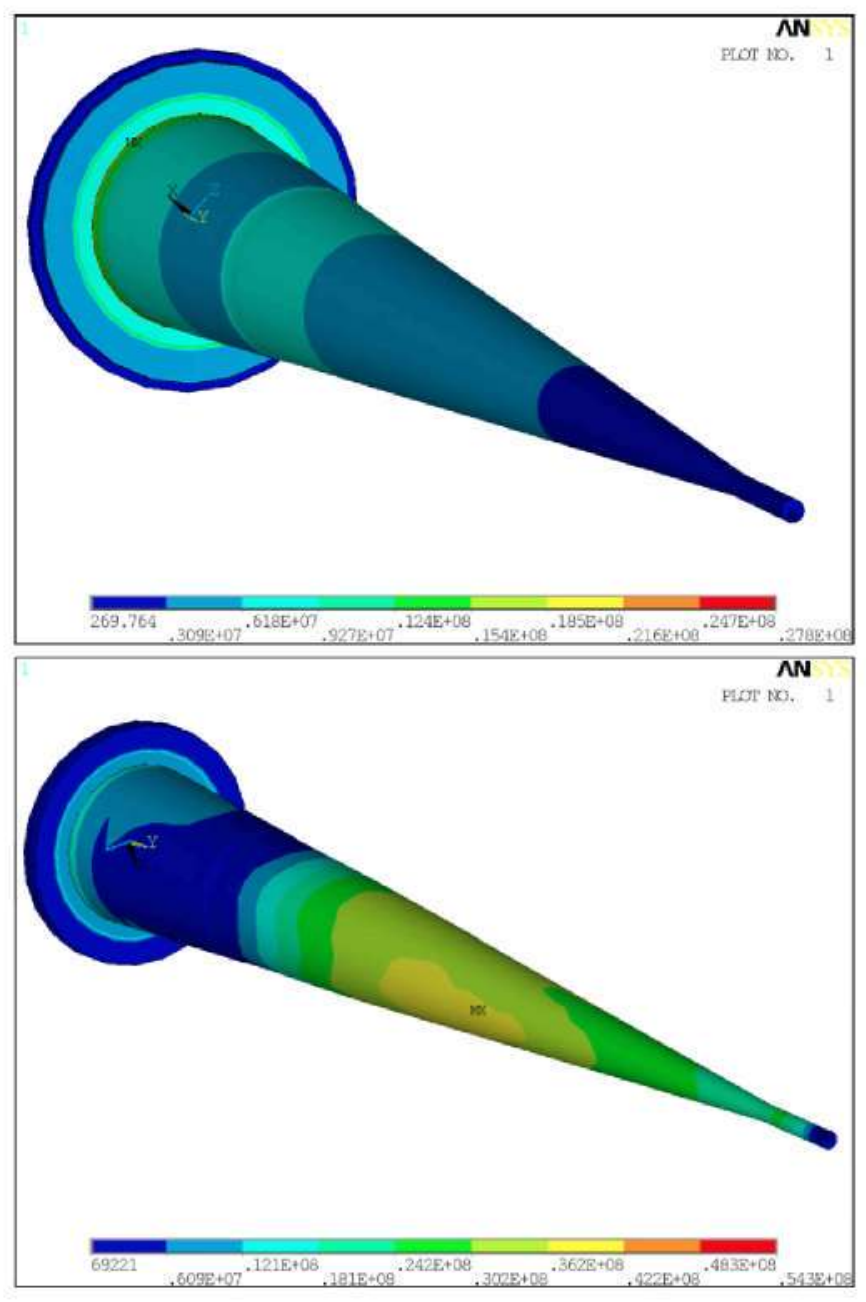

Fig. 2. Von Mises stress for pressure and gravity (top), and nominal beam (bottom).

at high velocity $(8.5 \mathrm{~m} / \mathrm{s})$ at the cone vertex and flows in counterbeam direction. In the geometry of the coolant channel that has been selected, the water flows between the internal cone and the external tube which is made of three cones with different slopes. The minimum width of the channel $(7 \mathrm{~mm})$ is achievable, taking account of manufacturing, assembly and mounting tolerances.

An inlet water temperature of $31^{\circ} \mathrm{C}$ is assumed since the minimum temperature at the heat exchanger is $27^{\circ} \mathrm{C}$. A flow rate of $30 \times 10^{-3} \mathrm{~m}^{3} / \mathrm{s}$ has been specified as adequate to minimize the required pressure. With this flow rate a pressure of $3.4 \times 10^{5} \mathrm{~Pa}$ at the inlet is appropriate to give adequate margin to prevent boiling. The calculated resulting temperature profile is shown in Fig. 1.

\subsection{Thermomechanical analysis}

The mechanical analysis has been performed for nominal conditions and also for different input parameters to determine the

Table 1

Maximum values of control parameters for beam error simulations.

\begin{tabular}{|c|c|c|c|c|}
\hline Error effect and value & Max. internal temperature $\left({ }^{\circ} \mathrm{C}\right)$ & Maximum stress $(P+Q)(\mathrm{MPa})$ & Maximum stress location & Max. displacement $\mathrm{x}$-axis (mm) \\
\hline Nominal & 131.25 & 54.3 & Peak power & 0.15 \\
\hline Shifted $15 \mathrm{~mm}$ & 148.73 & 87.7 & Tip & 2.18 \\
\hline Steered 6 mrad & 163.42 & 134.0 & Tip & 3.15 \\
\hline Defocused $30 \%$ & 117.90 & 42.4 & Peak power & 0.15 \\
\hline Focused $30 \%$ & 181.70 & 82.7 & Peak power & 0.16 \\
\hline
\end{tabular}




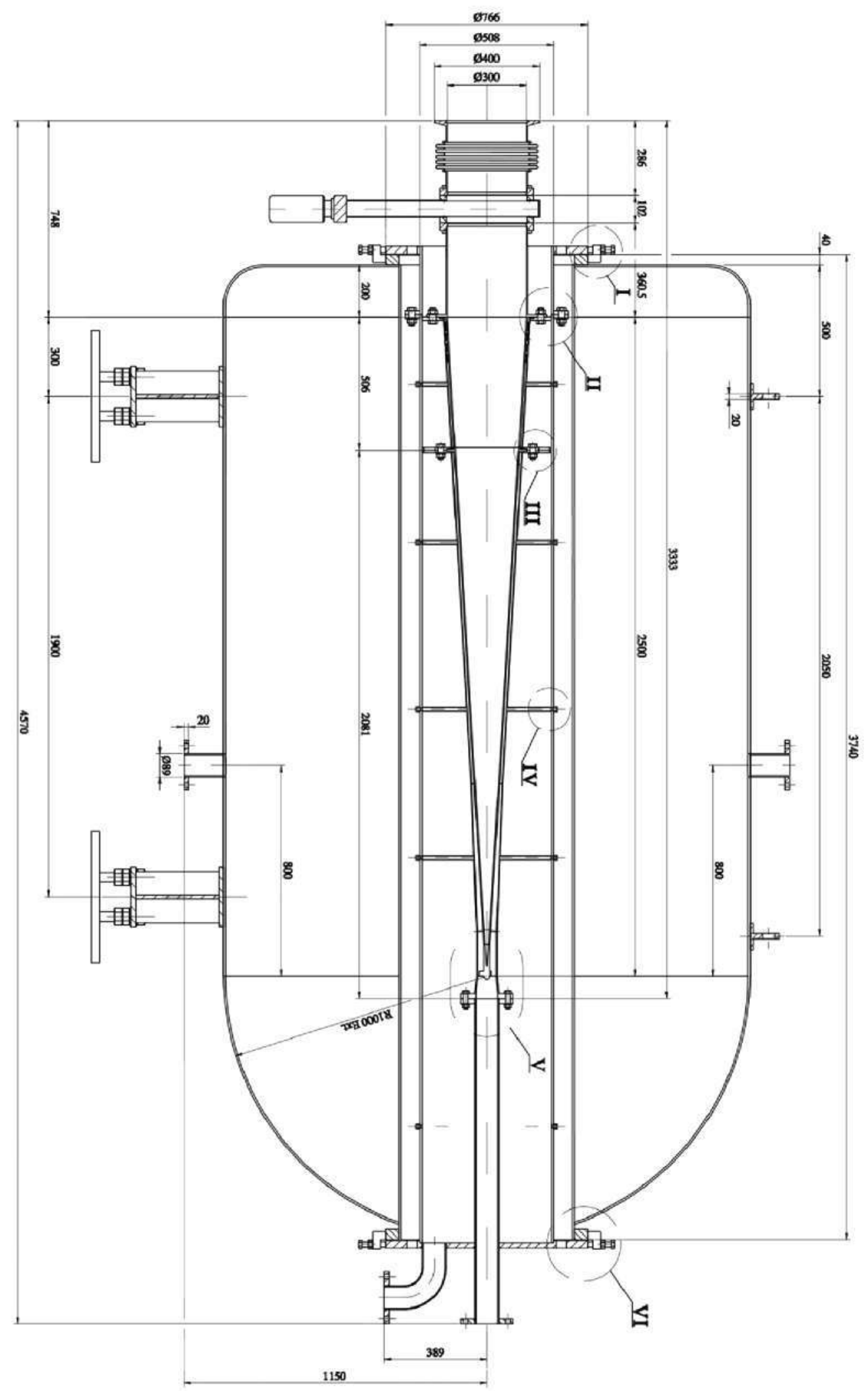

Fig. 3. Layout drafts of the beam dump assembly.

working range of the system. As the deuterons are stopped in the first $10 \mu \mathrm{m}$, surface energy deposition is assumed.

Along with the power density deposition, the loading hypothesized also includes the coolant pressure and gravity effects. The off normal conditions of the beam include a $30 \%$ variation in focusing, $15 \mathrm{~mm}$ offset and $6 \mathrm{mrad}$ angular deviation. The struc- ture shape and thickness have been chosen so that the mechanical response is optimized for all load conditions. The resulting values of $5 \mathrm{~mm}$ and $6.5 \mathrm{~mm}$ (see Fig. 1) arise from these design criteria.

The Design Standard applied to validate the model is the ASME code, section VIII, division II: Rules for construction of pressure 
vessels, alternative rules [6]. This ASME code defines the reference stress intensity strength of OFE-Cu value at $149{ }^{\circ} \mathrm{C}$ as $S_{\mathrm{m}}=34.5 \mathrm{MPa}$.

Apart from beam loading, the stresses are produced by the coolant pressure and gravity effects (Fig. 2, top). A pre-stressed calculation has been performed for buckling analysis, obtaining a first mode load multiplier value of 21 , which is considered safe enough based on previous designs.

When the beam hits the beam dump, the effects of the power deposition are added (Fig. 2, bottom). Table 1 summarizes the results for the nominal beam and different error effects. The maximum stress should be below $3 S_{\mathrm{m}}=103.5 \mathrm{MPa}$. This value is only exceeded at the tip of the cone when the beam steers 6 mrad off axis, so better material properties are needed in this zone for handling such an error.

\subsection{Beam dump layout}

Fig. 3 shows the assembly which includes the inner cone, the outer cone, the cylinder which supports both cones, the lower tank and the upper tank. The cylinder containing the two cones is referred to as "the cartridge". The cooling system connections are located at the rear of the beam dump. A straight pipe $(89 \mathrm{~mm}$ diameter) feeds water to the cooling channel between the two cones. The water changes direction at the end of the cone, returns through the space between the cylinder and the outer cone, and then exits at the back through a $90^{\circ}$ bend. A vacuum valve at the aperture of the cone can isolate the beam dump in case of need. Between this valve and the beam tube there is a bellow which allows small movements for aligning the beam dump. The water tank is divided in two parts to allow dismantling of the cartridge from the top using a remotely operated crane.

The instrumentation foreseen in the cartridge includes hydrophones and radiation monitors. The use of other diagnostics such as thermocouples and strain gages is difficult due to the space constraints and the radiation environment.

\section{Manufacturing process survey}

The most critical part of the beam dump is the internal cone which receives the deuteron beam.

The tentatively selected material is oxygen free copper UNS $\mathrm{N}^{\circ}$ C10200, but C10100 is also a candidate, depending on the importance of gas desorption, which shall be studied along with the vacuum system design team. It is very important that the raw material as well as the parts produced during the fabrication process maintain the very pure chemical composition, especially at the inner surface, because the activation and radiological studies would be invalid if new elements are added ( $\mathrm{Al}, \mathrm{Fe}$, etc.). If contamination occurs or is foreseen during the fabrication process, the influence on radiological behavior and corrosion resistance must be analyzed and/or a procedure for cleaning the affected surface to remove problem contaminants must be developed.

As far as possible, the beam dump should be manufactured as a single piece, at least the zone where most of the energy deposition takes place, between the vertex of the cone and the change in thickness. This will minimize any inhomogeneous thermal and mechanical properties of welded and weld-affected regions.

The vertex zone must have better mechanical properties than the rest of the beam dump because it will need to withstand higher thermal stresses on the inner spherical surface, and mechanical stresses due to bending at the outer surface. A value of 150 $200 \mathrm{MPa}$ yield strength would be desirable for this zone. The rest of the cone will need mechanical properties complying with ASME BPV code requisites for material UNS $\mathrm{N}^{\circ} \mathrm{C} 10200$, with yield strength of at least $68.9 \mathrm{MPa}$ at $20^{\circ} \mathrm{C}$ and $55.1 \mathrm{MPa}$ at $121^{\circ} \mathrm{C}$.
At the moment the preferred technology for manufacturing the inner cone seems to be electroforming of copper on a mandrel. Electron beam welding of truncated cones machined in a lathe has also been considered.

\section{Radiological protection analysis}

\subsection{Local shielding design}

The beam stop element is held inside a cartridge with water cooling. The cartridge is located inside a shielding water tank with an aperture for the incoming beam. The whole system is enclosed in a separated room (the beam dump cell) formed of $50 \mathrm{~cm}$ concrete walls. The beam tube penetrates into the cell through a $30 \mathrm{~cm}$ diameter hole directly facing the aperture of the beam stop. The building walls are $1.5 \mathrm{~m}$ concrete.

In order to keep the dose rate in the accelerator area below $12.5 \mu \mathrm{Sv} / \mathrm{h}$ after shutdown, a cylindrical lead plug is included at the beam dump entrance. The thickness of the plug is $20 \mathrm{~cm}$ and the radius is the same as that of the water tank. This disk will be made in two parts: a small plug which will be located outside the beam dump cell and will be placed inside the beam tube after shutdown, and the remaining lead crown which is inside the beam dump cell.

\subsection{Beam-on and beam-off radiation fields}

The slowing-down of deuterons from SRIM and the total neutron cross-section taken from the EAF library [7] have been used

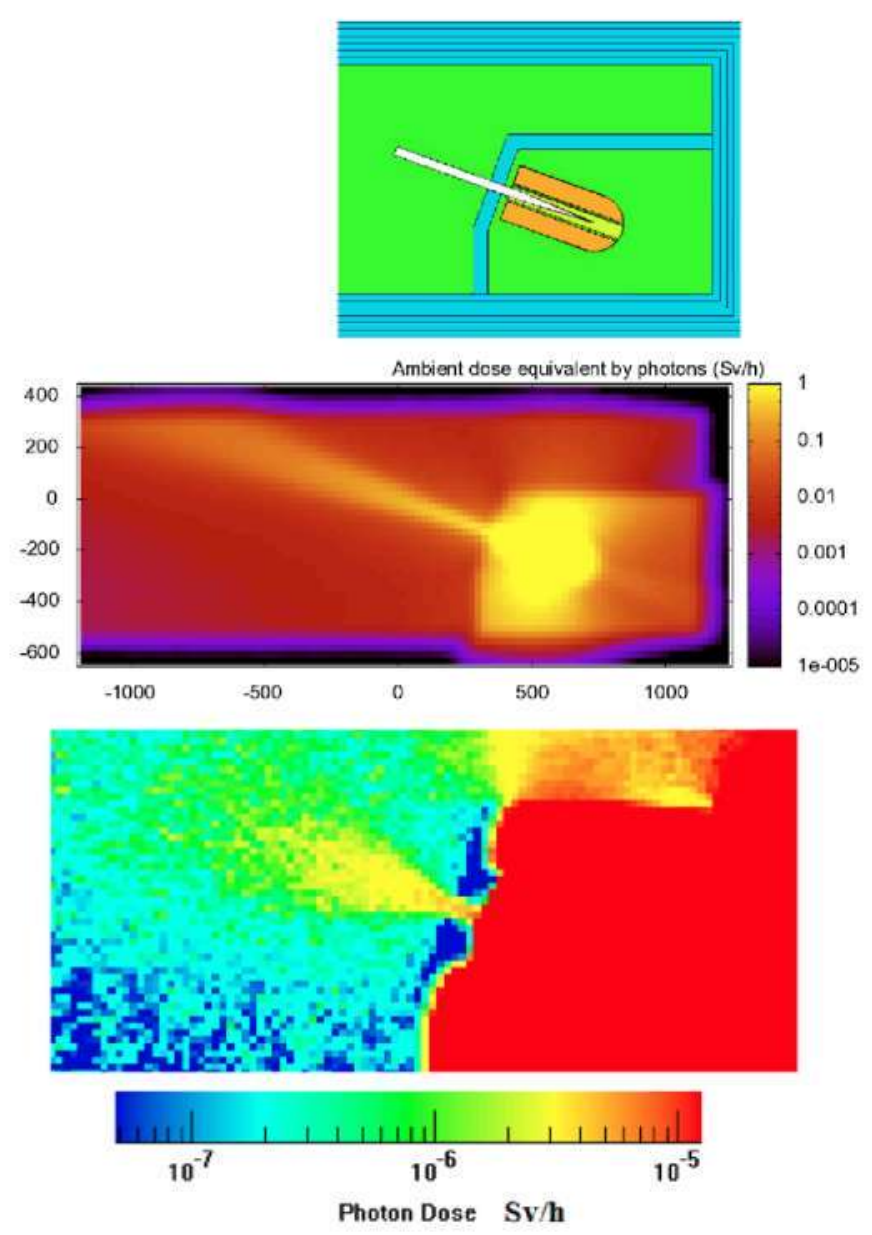

Fig. 4. Photons dose rates for beam-on (top) and beam-off (bottom) conditions. 
to calculate radiation fields. More details can be found in [8]. The local shielding solution for the beam dump has proven to effectively stop most of the neutrons coming from the beam stop and the photon source generated by neutron interactions with the shielding water (Fig. 4, top).

This shows that with the proposed lead plug the dose rates requirements in the accelerator vault after one day cooling are fulfilled (Fig. 4, bottom). The contribution of the lead plug activation to the dose rates was found to be negligible.

\section{Conclusions}

The present design for the IFMIF-EVEDA accelerator beam dump is based on a conical copper beam stop ( $2.5 \mathrm{~m}$ length, $30 \mathrm{~cm}$ diameter, and variable thickness $5-6.5 \mathrm{~mm}$ ). The cooling system is based on axial high velocity water flow. This design is compliant with the mechanical design rules during full power operation of the accelerator, with nominal beam and also considering possible beam errors. The radiological protection calculations demonstrate that doses during beam-on and beam-off phases are below limits with an adequate local shielding.

\section{Acknowledgement}

This work has been partially supported by the spanish Ministry of Science and Innovation under Project ENE2009-11230.

\section{References}

[1] A. Mosnier, A. Facco, A. Ibarxra, in: Proceedings of the European Particle Accelerator Conference, 2008, p. 3539.

[2] B. Brañas, D. Iglesias, F. Arranz, G. Barrera, N. Casal, M. García, et al., Fusion Eng. Des. 84 (2009) 509-513.

[3] B. Brañas, F. Arranz, G. Barrera, J.M. Gómez, A. Ibarra, D. Iglesias, et al., in: Proceedings of the European Particle Accelerator Conference, 2008, p. 259

[4] C. Oliver, B. Brañas, A. Ibarra, I. Podadera, N. Chauvin, A. Mosnier, et al. in: Proceedings of the European Particle Accelerator Conference, 2008, p. 3041 .

[5] SRIM. Stopping Range of lons in Matter. 〈http://www.srim.org〉.

[6] ASME Vill. Div. 2. Pressure vessels.

[7] R.A. Forrest, The European Activation File: EAF-2007 Deuteron and Proton Induced Cross Section Libraries.

[8] J. Sanz, M. García, P. Sauvan, D. López, C. Moreno, A. Ibarra, et al., J. Nucl. Mater. (2008), doi:10.1016/j.jnucmat.2008.12.277.

[9] T.H. Van Hagan, D.W. Doll, J.D. Schneider, F.R. Spinos, Design of an ogive-shaped beamstop,in: Proceedings of the LINAC '98. Chicago, IL, 1998. 